\title{
Weakly relativistic dielectric tensor for arbitrary wavenumbers
}

\author{
Francesco Volpe* \\ Max-Planck-Institut für Plasmaphysik, D-17491 Greifswald, Germany
}

(Dated: October 16, 2007)

\begin{abstract}
The validity of Shkarofsky's dielectric tensor is extended by taking the strictly weakly relativistic limit and removing, when possible, assumptions on the wavenumbers along and across the ambient magnetic field, $k_{\|}$and $k_{\perp}$. An approximation of the time integral is retained, but is shown to be valid under more benign assumptions than those of quasi-perpendicular incidence and small Larmor radius. The increased generality with respect to $k_{\|}$permits to handle cases of comparable Doppler and relativistic widths of electron cyclotron resonances. The tensor also suits Bernstein waves, as it captures both their natural large $k_{\perp}$ and the finite $k_{\|}$that is typical of some mode conversions, or acquired as a consequence of the large $k_{\perp}$ when propagating in curved magnetic fields. Finally, relativistic corrections to the optimal angle for the ordinary-extraordinary-Bernstein mode conversion are presented.
\end{abstract}




\section{INTRODUCTION}

The relativistic dielectric tensor $\epsilon$ was first obtained by Trubnikov by solving the relativistic Vlasov equation in terms of a small perturbation to a maxwellian distribution ${ }^{1}$. A more treatable weakly relativistic approximation was then derived by Dnestrovskii for propagation perpendicular to the magnetic field ${ }^{2}$. Later, this was extended by Shkarofsky to quasi-perpendicular incidence, such that ${ }^{3}$

$$
\begin{aligned}
N_{\|} & \ll \beta_{T} \ll 1, \\
\left|N_{\|}\left(2 \delta-N_{\|}^{2}\right)\right| & \ll \beta_{T}^{2} .
\end{aligned}
$$

Here $N_{\|}$is the parallel refractive index, $\beta_{T}^{2}=k_{B} T / m c^{2}$ the squared thermal velocity in $c$ units and $\delta=\left(\omega-n \omega_{c}\right) / \omega$ indicates the distance from the $n$-th cyclotron harmonic in dimensionless units.

In addition to making these hypotheses on $N_{\|}$, Shkarofsky's and most subsequent weakly relativistic formulations also expand $\epsilon$ as a power series in the (small) Larmor radius parameter $^{3-11}$

$$
\lambda=\left(\frac{N_{\perp} \beta_{T}}{Y}\right)^{2}<1
$$

or take the opposite, asymptotic limit $\lambda>1^{12,13}$. Here $Y=\omega_{c} / \omega$ is the dimensionless magnetic field. These series are formally correct, in the sense that, if they converge, they do so to an exact result. Convergence is ensured in one case by $\lambda<1$ and in the other by $\lambda>1$, so that, for any $\lambda$, one can always choose a converging expansion of $\epsilon$ in cyclotron harmonics. In practice, however, these series will be truncated and provide approximate results, valid in the $\lambda \ll 1$ or $\lambda \gg 1$ limit, respectively. The fewer the terms retained in the sum, the smaller is the range of validity in $\lambda$. Of course expressions, available in literature, which are already truncated, have a reduced range of validity. All these series expansions and truncated sums are summarized in Table I, and some of them were reviewed in ${ }^{14}$. In Table I we also note that not the whole $\epsilon$ but only an electrostatic approximation is considered in $^{11-13}$. Apart from a very general but numerically expensive series in a 4D space multiplied by a sum in a $2 \mathrm{D}$ space and a double combinatoric $\mathrm{sum}^{15}$, some approximations may not be adequate for electron Bernstein waves, as these waves are characterised by $\lambda \gtrsim 1$ and are excited/detected at finite $N_{\|}^{16-19}$ or tend to develop finite $N_{\|}$anyway, including values 
$N_{\|} \geq 1^{19,20}$. Finite values of $N_{\|}$, of the order of $\beta_{T}$, are also encountered in ion Bernstein waves, for which they were treated numerically with the aid of root finders ${ }^{21}$.

Electromagnetic electron cyclotron waves propagating at intermediate angles relative to the magnetic field may also have $N_{\|} \approx \beta_{T}$. In this case the two mechanisms resolving the electron cyclotron emission/absorption line coexist and the fully relativistic $\epsilon$, which embodies both the Doppler broadening and relativistic mass gain, should be invoked. However, because it is very complicated and not in a closed form, an analytic semi-relativistic form of $\epsilon$ valid for arbitrary values of $N_{\|}$and $N_{\perp}$ is derived in the present work. Note that simplified 1D relativistic descriptions are available, for example for reflectometry ${ }^{22}$. However, modelling in 3D requires either the fully ${ }^{1}$ or weakly ${ }^{3-8,8-13,15}$ relativistic dielectric tensor. Here a compromise is proposed between the generality of the full tensor and the ease of calculation of the weakly relativistic one.

Two well-known weakly relativistic approximations ${ }^{36}$ are compared in Sec.II and the most rigorous is adopted. Only the strict weakly relativistic limit $\beta_{T} \ll 1$ is taken here. Unlike other works, the treatment is not restricted to $N_{\|} \ll \beta_{T}$, that would help isolating relativistic effects from Doppler ones, but is not the most general assumption. A cyclotron harmonic expansion is operated in Sec.III. The arbitrariness of $N_{\|}$leads to a further generalization of the generalized Shkarofsky functions and to new, $N_{\|}$-dependent terms in $\epsilon$. In Sec.IV, this new mildly relativistic expression is compared with earlier results. Both warm and Shkarofsky formulas, in particular, are reobtained as limiting cases. This implies the new tensor to be valid for both oblique, Doppler-dominated injection and perpendicular, relativistically broadened absorption.

Sec.IV also shows that the generalization introduced in Sec.III is equivalent to perturb the arguments of the Bessel functions, which cannot be factored out of the integral anymore. Numerical results in Sec.V illustrate the broader domain of applicability of the new expression with respect to $N_{\|}$and $N_{\perp}$, at a modest computational extra cost. Finally, some calculations pertinent to Sec.III are reported in Appendix. 


\section{WEAKLY RELATIVISTIC LIMIT}

The steady-state solution of the linearized Vlasov-Maxwell problem for a uniformly magnetized plasma is ${ }^{1,8}$ :

$$
\epsilon_{i j}=\delta_{i j}+\frac{i X}{\beta_{T}^{2}} \int_{0}^{\infty} d \tau I_{i j}(\tau)
$$

where $X=\omega_{p}^{2} / \omega^{2}$ is the non-dimensional density and $\tau$ the time renormalized to the wave period $\omega^{-1}$. Apart from a factor $n Y, \tau$ coincides with the gyrophase and, for practical uses, it is sufficient to integrate Eq.4 over a time $\tau$ long enough for the wave-particle interaction to take place, bearing in mind that correlation is destroyed after a certain number of gyroperiods.

The function $I_{i j}(\tau)$ is defined as an integral over the normalized momentum $\mathbf{u}=\mathbf{p} / m c$ :

$$
I_{i j}=T_{j k}^{(1)} \int \frac{d^{3} \mathbf{u}}{\gamma} u_{i} u_{k} f(\mathbf{u}) \exp (i \gamma \tau-i \mathcal{N} \cdot \mathbf{u})
$$

where $\gamma=\sqrt{1+\mathbf{u}^{2}}$ is the Lorentz factor and the convention of implicitly summing on repeated indices is adopted. The tensor

$$
T^{(1)}=\left(\begin{array}{ccc}
\cos Y \tau & -\sin Y \tau & 0 \\
\sin Y \tau & \cos Y \tau & 0 \\
0 & 0 & 1
\end{array}\right)
$$

defines a rotation of the reference frame at an angular frequency equal to the (normalized) gyrofrequency $Y=\omega_{c} / \omega$ around the field-aligned axis $z$. The remaining axes are chosen to yield $\mathbf{N}=\left(N_{\perp}, 0, N_{\|}\right)$. Finally,

$$
\begin{aligned}
& \mathcal{N}_{x}=\frac{N_{\perp}}{Y} \sin Y \tau \\
& \mathcal{N}_{y}=\frac{N_{\perp}}{Y}(\cos Y \tau-1) \\
& \mathcal{N}_{z}=N_{\|} \tau
\end{aligned}
$$

and, for a thermal relativistic plasma, the distribution function equals

$$
f(\mathbf{u})=\frac{\exp \left(-\gamma \beta_{T}^{-2}\right)}{4 \pi \beta_{T}^{2} K_{2}\left(\beta_{T}^{-2}\right)}
$$

where $K_{2}$ is the modified Bessel function of the second kind of order 2, also known as MacDonald function ${ }^{23}$. 
All parameters and independent variables in integral Eq.5 are real, apart from the refractive index components which are complex, with $\Re\left(N_{\perp}\right) \geq 0$.

Integration over momenta gives ${ }^{1}$ :

$$
I_{i j}=\frac{\beta_{T}^{-2}}{K_{2}\left(\beta_{T}^{-2}\right)}\left[T_{i j}^{(1)} \frac{K_{2}\left(R^{1 / 2}\right)}{R}-T_{i j}^{(2)} \frac{K_{3}\left(R^{1 / 2}\right)}{R^{3 / 2}}\right]
$$

where

$$
\begin{aligned}
R & =b^{2}+\mathcal{N}^{2}=\frac{1}{\beta_{T}^{4}}-\frac{2 i}{\beta_{T}^{2}} \tau+2 \frac{N_{\perp}^{2}}{Y^{2}}(1-\cos Y \tau)+\left(N_{\|}^{2}-1\right) \tau^{2} \\
b & =\frac{1}{\beta_{T}^{2}}-i \tau
\end{aligned}
$$

and $T_{i j}^{(2)}=(-)^{j+1} \mathcal{N}_{i} \mathcal{N}_{j}=\mathcal{N}_{i} T_{j k}^{(1)} \mathcal{N}_{k}$ is the tensor

$$
T^{(2)}=\left(\begin{array}{ccc}
\mathcal{N}_{x}^{2} & \mathcal{N}_{x} \mathcal{N}_{y} & \mathcal{N}_{x} \mathcal{N}_{z} \\
-\mathcal{N}_{x} \mathcal{N}_{y} & -\mathcal{N}_{y}^{2} & -\mathcal{N}_{y} \mathcal{N}_{z} \\
\mathcal{N}_{x} \mathcal{N}_{z} & \mathcal{N}_{y} \mathcal{N}_{z} & \mathcal{N}_{z}^{2}
\end{array}\right)
$$

\section{A. Weakly relativistic limit of Eq.11}

Eq.4 with $I_{i j}$ as in Eq.11, is known as the second of Turbnikov's formulas. The essential step in its weakly relativistic approximations $s^{3,7,13}$ is to assume the argument $R$ of the modified Bessel functions to be large.

Clearly, from Eq.12, $R$ is a function of the independent variables $\beta_{T}, N_{\|}$and $N_{\perp}$ and at this stage the $\beta_{T} \rightarrow 0$ limit can be taken without implications for the other (orthogonal) directions $N_{\|}$and $N_{\perp}$. In particular, it is not necessary to assume small Larmor radii (Eq.3) and/or quasi-perpendicular incidence (Eq.1). The strict mildly relativistic limit $\beta_{T} \ll 1$ is sufficient, although not necessary, for $R \gg 1$. In turn, this justifies the asymptotic $\operatorname{expansion}^{23}$

$$
K_{\nu}\left(R^{1 / 2}\right) \simeq \sqrt{\frac{\pi}{2}} \frac{e^{-R^{1 / 2}}}{R^{1 / 4}}\left\{1+\sum_{n=1}^{\infty} \frac{\prod_{p=1}^{n}\left[4 \nu^{2}-(2 p-1)^{2}\right]}{n !\left(8 R^{1 / 2}\right)^{n}}\right\}
$$

where, for the case of interest, $\nu=2$ or 3 .

With this, Eq.11 becomes:

$$
\begin{aligned}
I_{i j} & =\frac{e^{\beta_{T}^{-2}}}{\beta_{T}^{3}+\frac{15}{8} \beta_{T}^{5}+\frac{105}{128} \beta_{T}^{7}} e^{-R^{1 / 2}} Q_{i j} \\
Q_{i j} & =\frac{T_{i j}^{(1)}}{R^{5 / 4}}\left(1+\frac{15}{8 R^{1 / 2}}+\frac{105}{128 R}\right)-\frac{T_{i j}^{(2)}}{R^{7 / 4}}\left(1+\frac{35}{8 R^{1 / 2}}+\frac{945}{128 R}\right)
\end{aligned}
$$


The square root at exponent here, $e^{-R^{1 / 2}}$, poses a major difficulty for the integration of Eq.4. Factorizing $e^{-R^{1 / 2}} \simeq e^{-b} e^{-\mathcal{N}^{2} / 2 b}$ (see Eq.12) helps to avoid that difficulty. For this, it must be $\mathcal{N}^{2} \ll\left|b^{2}\right|$, where || denotes the modulus of a complex number.

Besides simplifying Eq.16, the $\mathcal{N}^{2} \ll\left|b^{2}\right|$ limit also modifies Eq.17 as follows:

$$
Q_{i j}=T_{i j}^{(1)} b^{-9 / 2}\left(b^{2}+\frac{15}{8} b+\frac{105}{128}-\frac{5}{4} \mathcal{N}^{2}\right)-T_{i j}^{(2)} b^{-11 / 2}\left(b^{2}+\frac{35}{8} b+\frac{945}{128}-\frac{7}{4} \mathcal{N}^{2}\right)
$$

The limit in question is a small $\beta_{T}$ limit which also interests $\mathcal{N}$ and thus the refractive index components. However, here below it is shown to be less restrictive than Eq.1.

From the definitions 12-13, the inequality writes:

$$
2 \frac{N_{\perp}^{2}}{Y^{2}}(1-\cos Y \tau)+N_{\|}^{2} \tau^{2} \ll \frac{1}{\beta_{T}^{4}}+\tau^{2}
$$

The smallness -at any time $\tau$ - of the first term on the left hand side requires $\frac{N_{\perp}^{2}}{Y^{2}} \ll \frac{1}{\beta_{T}^{4}}$ i.e. relatively small Larmor radii $\left(\lambda \ll \frac{1}{\beta_{T}^{2}}<\right)$, although not as small as in the conventional finite Larmor radius (FLR) limit $(\lambda \ll 1)$. For example, for electromagnetic waves of $N_{\perp} \leq 1$, it is sufficient to meet a requirement on the magnetic field, $Y \gg \beta_{T}^{2}$, which is fulfilled in most magnetized laboratory plasmas. For Bernstein waves (reaching the highest perpendicular refractive index, $N_{\perp} \approx \frac{Y}{\beta_{T}}$, such that $\lambda \approx 1$ ) the requirement is simply $\beta_{T} \ll 1$, which is being assumed through this weakly relativistic treatment anyway.

The second term on the left hand side of Eq.19, $N_{\|}^{2} \tau^{2}$, should in principle be much smaller than the right hand side. This is always the case if $N_{\|} \leq 1$, at any time $\tau$. If $N_{\|}>1$ the condition is violated from time $\tau_{1}=\frac{1}{\beta_{T}^{2}} \frac{1}{\sqrt{N_{\|}^{2}-1}}$ onwards. However, the wave-particle interaction weakens as time progresses and the decay is particularly rapid in the $N_{\|}>1$ case. This is because in that case, apart from an oscillation due to the finite $N_{\perp}, R$ is a growing function of $\tau$, and the integrand $I_{i j}$ of Eq.4 decays exponentially with $R$, as illustrated by Eq.16. To fix the ideas, the wave-particle interaction can be considered negligible when $e^{-R^{1 / 2}}$ reaches a thousandth of its initial value $e^{-\beta_{T}^{-2}}$. This occurs at $\tau_{2}=\sqrt{\frac{49+14 / \beta_{T}^{2}}{N_{\|}^{2}-1}}$. Therefore, it is sufficient to require the wave-particle interaction to become negligible before the condition 19 is formally violated. This translates into the condition $\tau_{2}<\tau_{1}$, which is equivalent to $\beta_{T}<0.24$, or $T_{e}<30 \mathrm{keV}$. In turn, this should already be guaranteed by $\beta_{T} \ll 1$. More generally, the condition is $\beta_{T}^{2}<\frac{\sqrt{2}-1}{\ln D}$. Here, to fix the ideas, strong damping was defined as damping by a factor $D=1000$. 
Recapitulating, Eq.19 is valid for any $N_{\|}$, provided $\frac{N_{\perp}}{Y} \ll \frac{1}{\beta_{T}^{2}}$ and $\beta_{T} \ll 1$ or, equivalently, $\beta_{T} \ll \min \left\{1, \sqrt{Y / N_{\perp}}\right\}$. Under these circumstances $e^{-R^{1 / 2}} \simeq e^{-b} e^{-\mathcal{N}^{2} / 2 b}$, which simplifies the

integration of Eq.4. $\frac{N_{\perp}}{Y} \ll \frac{1}{\beta_{T}^{2}}$ is indeed a FLR condition, as it may be rewritten as $\lambda \ll \frac{1}{\beta_{T}^{2}}$, but less stringent than Eq.3, as it includes higher order FLR corrections compared to $\lambda \ll 1$.

Note that Eq.19 is an independent requirement not related to Eqs.1-2, which are not invoked here. All these equations impose an upper limit to $N$ components, although the limit scales like powers of $\beta_{T}$ in Eqs.1-2 and like an inverse power in Eq.19. In this respect Eq.19 is more similar to the small Larmor radius condition Eq.3, but is less restrictive for $N_{\perp}$, thank to the weakly relativistic limit.

\section{B. Weakly relativistic limit of the integrand in Eq.5}

For an alternative derivation of Eqs.16-18, $\gamma$ can be Taylor-expanded up to the second order in $u$ in Eq.5, which takes the form:

$$
I_{i j}=\frac{e^{i \tau}}{\left(2 \pi \beta_{T}^{2}\right)^{3 / 2}\left(1+\frac{15}{8} \beta_{T}^{2}\right)} T_{j k}^{(1)} \int d^{3} \mathbf{u} u_{i} u_{k}\left(2-u_{x}^{2}-u_{y}^{2}-u_{z}^{2}\right) \mathcal{E}_{x} \mathcal{E}_{y} \mathcal{E}_{z} / 2
$$

where

$$
\mathcal{E}_{x}=\exp \left[-i \mathcal{N}_{x} u_{x}+\frac{u_{x}^{2}}{2}\left(i \tau-\beta_{T}^{-2}\right)\right]
$$

and equivalent definitions apply to $\mathcal{E}_{y}$ and $\mathcal{E}_{z}$.

After substituting Eq.9, one can recognize in $\mathcal{E}_{z}$ the term $-i N_{\|} u_{\|} \tau$ responsible for Doppler broadening. The source of weakly relativistic broadening $i u^{2} \tau / 2$ dominates over it if $N_{\|} \ll u$, but for the sake of generality and to cope with $N_{\|} \approx u$, both terms are retained here.

The main advantage of Eq.20 over Eq.5 is that the parallel and perpendicular degree of freedom, which were previously coupled by $\gamma$, are now decoupled. The approximation of $\gamma^{-1}$ with a sum and of the exponent with a product of functions of $u_{x}, u_{y}$ or $u_{z}$ only, ease the integration over $\mathbf{u}$. In fact, this reduces to a sum of products of integrals of type:

$$
\int_{-\infty}^{\infty} d u_{x} e^{-i \mathcal{N}_{x} u_{x}-b u_{x}^{2} / 2}=\sqrt{\frac{2 \pi}{b}} e^{-\mathcal{N}_{x}^{2} / 2 b}
$$

and its derivatives up to the 4 th order in $\mathcal{N}_{x}$. Similar integrals in $u_{y}$ and $u_{z}$, as well as their derivatives, are also involved. Note that in the integrals above the real part of $b$ is positive. After some algebra, the same result as in Eq.16 is obtained, but with different 
coefficients for Eq.18:

$$
Q_{i j}=T_{j i}^{(1)} b^{-9 / 2}\left(b^{2}-\frac{5}{2} b+\frac{\mathcal{N}^{2}}{2}\right)-T_{j i}^{(2)} b^{-11 / 2}\left(b^{2}-\frac{7}{2} b+\frac{\mathcal{N}^{2}}{2}\right)
$$

This is because, although physically more explicit, the second method is based on the exact integration of an approximated integrand (Eq.20). Therefore, it is only valid in the limit in which the approximated distribution and wave-particle interaction are plausible. Although not maxwellian, neither classically nor relativistically, the distribution $f$ used in Eq.20 is correct in a semi-relativistic sense and is correctly normalized, i.e. the approximation conserves the number of particles. However, the description of the wave-particle interaction in Eq.20 is correct only at the leading orders in $b$. For these reasons, we will adopt for the remainder the more rigorous Eq.18, resulted from the Taylor approximation of the exact integral, Eq.11.

\section{EXPANSION IN CYCLOTRON HARMONICS}

First of all, it is convenient to change variables and absorb the thermal velocity in the definitions of time and cyclotron frequency:

$$
t=\beta_{T}^{2} \tau \quad y=Y / \beta_{T}^{2}
$$

The contributions of individual cyclotron harmonics to Eq.16 can be separated from each other by rewriting as follows a term involved in $e^{-R^{1 / 2}}$ :

$$
\exp \left[\frac{\lambda \cos y t}{1-i t}\right]=\sum_{p=0}^{\infty} \frac{1}{p !}\left[\frac{\lambda}{1-i t} \frac{e^{i y t}+e^{-i y t}}{2}\right]^{p}
$$

with the double series restricted to even values of $m+n$.

The binomial theorem transforms Eq.25 in

$$
\exp \left[\frac{\lambda \cos y t}{1-i t}\right]=\sum_{p=0}^{\infty} \sum_{q=0}^{\infty}\left[\frac{\lambda}{2(1-i t)}\right]^{p+q} \frac{e^{i(p-q) y t}}{p ! q !}
$$

Finally, considerations about how to fill the first quadrant $p>0, q>0$, with sums over $m=p+q$ and $n=p-q$, lead to:

$$
\exp \left[\frac{\lambda \cos y t}{1-i t}\right]=\sum_{n=-\infty}^{\infty} \sum_{m=|n|}^{\infty}\left[\frac{\lambda}{2(1-i t)}\right]^{m} \frac{e^{i n y t}}{\left(\frac{m+n}{2}\right) !\left(\frac{m-n}{2}\right) !}
$$


With this substitution, Eqs.4 and 16 yield:

$$
\begin{aligned}
\epsilon_{i j} & =\delta_{i j}+\frac{i X}{\beta_{T}^{5}+\frac{15}{8} \beta_{T}^{7}} \sum_{n=-\infty}^{\infty} \sum_{m=|n|}^{\infty} \frac{(\lambda / 2)^{m} \mathcal{Q}_{m n, i j}}{\left(\frac{m+n}{2}\right) !\left(\frac{m-n}{2}\right) !} \\
\mathcal{Q}_{m n, i j} & =\int_{0}^{\infty} \frac{d t}{\beta_{T}^{2}} \frac{Q_{i j}}{(1-i t)^{m}} \exp \left[\frac{i t}{\beta_{T}^{2}}(1-n Y)-\frac{\lambda+N_{\|}^{2} t^{2} / 2 \beta_{T}^{2}}{1-i t}\right],
\end{aligned}
$$

where, as above, the summation is restricted to even values of $m+n$. All integrals of type 29 can be reconducted to generalized Shkarofsky functions ${ }^{24-26}$

$$
\mathcal{F}_{q, r}(z, a)=-i \int_{0}^{\infty} \frac{(i t)^{r}}{(1-i t)^{q}} \exp \left[i z t-\frac{a t^{2}}{1-i t}\right] d t
$$

but of shifted arguments that will be omitted for brevity:

$$
\mathcal{F}_{q, r}=\mathcal{F}_{q, r}(n)=\mathcal{F}_{q, r}\left(\frac{1-n Y}{\beta_{T}^{2}}-\lambda, \frac{N_{\|}^{2}}{2 \beta_{T}^{2}}-\lambda\right),
$$

Unless necessary for disambiguation, also the harmonic number will be dropped. In fact, most $\mathcal{F}_{q, r}$ hereafter refer to the $n$-th harmonic, except for some sums and differences on side-harmonics, coincisely referred to as:

$$
\mathcal{F}_{q, r}^{ \pm, p}=\mathcal{F}_{q, r}(n+p) \pm \mathcal{F}_{q, r}(n-p)
$$

Further, generalized Shkarofsky functions of $r \neq 0$ are related to standard Shkarofsky functions of $r=0$ by derivatives or integration by $\operatorname{parts}^{24}$,

$$
\begin{aligned}
\mathcal{F}_{q}^{\prime}=\frac{\partial \mathcal{F}_{q}}{\partial z} & =\mathcal{F}_{q, 1}=\mathcal{F}_{q}-\mathcal{F}_{q-1} \\
\mathcal{F}_{q}^{\prime \prime}=\frac{\partial^{2} \mathcal{F}_{q}}{\partial z^{2}} & =\mathcal{F}_{q, 2}=\mathcal{F}_{q}-2 \mathcal{F}_{q-1}+\mathcal{F}_{q-2}
\end{aligned}
$$

With these substitutions and after lengthy but straightforward calculations partly reported in appendix, one finds:

$$
\begin{aligned}
\mathcal{Q}_{m n, 11} & =i G_{m n}^{+}+i a_{+} \mathcal{F}_{m+\frac{9}{2}}-\frac{i}{4} \frac{N_{\perp}^{2}}{Y^{2}}\left[2 b_{l} \mathcal{F}_{m+\frac{l}{2}}+b_{+} \mathcal{F}_{m+\frac{11}{2}}^{+, 1}\right] \\
\mathcal{Q}_{m n, 22} & =i G_{m n}^{+}+i a_{+} \mathcal{F}_{m+\frac{9}{2}}-i \frac{N_{\perp}^{2}}{Y^{2}}\left[b_{l}\left(\mathcal{F}_{m+\frac{l}{2}}^{+, 1}-\frac{3}{2} \mathcal{F}_{m+\frac{l}{2}}\right)+b_{+}\left(\mathcal{F}_{m+\frac{11}{2}}^{+, 2}-\frac{7}{4} \mathcal{F}_{m+\frac{11}{2}}^{+, 1}+2 \mathcal{F}_{m+\frac{1}{2}} 3\right) \sigma_{j}\right) \\
\mathcal{Q}_{m n, 12} & =G_{m n}^{-}-\frac{1}{4} \frac{N_{\perp}^{2}}{Y^{2}}\left[2 b_{l} \mathcal{F}_{m+\frac{9}{2}}^{-, 1}+b_{+}\left(2 \mathcal{F}_{m+\frac{11}{2}}^{-, 2}-\mathcal{F}_{m+\frac{11}{2}}^{-, 1}\right)\right] \\
\mathcal{Q}_{m n, 13} & =-\frac{i}{2} \frac{N_{\perp}}{Y} \frac{N_{\|}}{\beta_{T}^{2}}\left(b_{l} \mathcal{F}_{m+\frac{l}{2}}^{-, 1}+b_{+} \mathcal{F}_{m+\frac{11}{2}}^{-, 2}\right)^{\prime} \\
\mathcal{Q}_{m n, 23} & =\frac{1}{2} \frac{N_{\perp}}{Y} \frac{N_{\|}}{\beta_{T}^{2}}\left[b_{l}\left(\mathcal{F}_{m+\frac{l}{2}}^{+, 1}-2 \mathcal{F}_{m+\frac{l}{2}}\right)^{\prime}+b_{+}\left(\mathcal{F}_{m+\frac{11}{2}}^{+, 2}-2 \mathcal{F}_{m+\frac{11}{2}}^{+, 1}+2 \mathcal{F}_{m+\frac{11}{2}}\right)^{\prime}\right] \\
\mathcal{Q}_{m n, 33} & =i\left(a_{l} \mathcal{F}_{m+\frac{l}{2}}+a_{+} \mathcal{F}_{m+\frac{9}{2}}^{+, 1}\right)+i \frac{N_{\|}^{2}}{\beta_{T}^{4}}\left(b_{l} \mathcal{F}_{m+\frac{l}{2}}+b_{+} \mathcal{F}_{m+\frac{11}{2}}^{+, 1}\right)^{\prime \prime}
\end{aligned}
$$


where

$$
G_{m n}^{ \pm}=\frac{1}{2}\left(a_{l} \mathcal{F}_{m+\frac{l}{2}}^{ \pm, 1}+a_{+} \mathcal{F}_{m+\frac{9}{2}}^{ \pm, 2}\right)+\frac{1}{4} \frac{N_{\perp}^{2}}{Y^{2}}\left(b_{l} \mathcal{F}_{m+\frac{l}{2}}^{ \pm, 2}+b_{+} \mathcal{F}_{m+\frac{11}{2}}^{ \pm, 3}\right),
$$

and $\mathcal{Q}_{m n, 21}=-\mathcal{Q}_{m n, 12}, \mathcal{Q}_{m n, 31}=\mathcal{Q}_{m n, 13}, \mathcal{Q}_{m n, 32}=-\mathcal{Q}_{m n, 23}$. It is worth reminding that here Shkarofsky functions $\mathcal{F}_{q, r}$ have shifted arguments like in Eq.31.

The convention of summing over repeated indices is adopted; scalar products involve the coefficients:

$$
\begin{aligned}
\left(a_{5}, a_{7}, a_{9}\right) & =\frac{5}{2} e^{-\lambda} \beta_{T}^{3}\left[\left(\frac{2}{5}+\frac{N_{\|}^{2}}{2}\right),\left(\frac{3}{4} \beta_{T}^{2}-N_{\|}^{2}\right),\left(\frac{N_{\|}^{2}}{2}-\lambda \beta_{T}^{2}\right)\right] \\
\left(b_{7}, b_{9}, b_{11}\right) & =\frac{7}{2} e^{-\lambda} \beta_{T}^{5}\left[\left(\frac{2}{7}+\frac{N_{\|}^{2}}{2}\right),\left(\frac{5}{4} \beta_{T}^{2}-N_{\|}^{2}\right),\left(\frac{N_{\|}^{2}}{2}-\lambda \beta_{T}^{2}\right)\right], \\
a_{+} & =\frac{5}{4} \lambda e^{-\lambda} \beta_{T}^{5} \\
b_{+} & =\frac{7}{4} \lambda e^{-\lambda} \beta_{T}^{7}
\end{aligned}
$$

Components of order $\beta_{T}^{4}$ were removed from the square brackets in Eqs.42-43, but those of order $\beta_{T}^{2}$ and, for consistency, of order $\beta_{T}^{2} \lambda$, were retained. The latter are formally of order $\beta_{T}^{4}$. Nevertheless, they also involve $N_{\perp}$, and neglecting them indirectly implies a restriction on the Larmor radius parameter that actually, for Bernstein waves, can evaluate $\lambda \lesssim 1$ even when $\beta_{T} \ll 1$. For the sake of generality, $N_{\|}^{2}$ has also been retained in all the components, both when it appears along with a number of order 1 , as well as with $\beta_{T}^{2}$ or $\lambda \beta_{T}^{2}$.

The $\lambda$-dependence of the revised generalized Shkarofsky functions is the sign that dispersion depends on the Larmor radius, in agreement with fully relativistic Eqs.4-5. The shift of $\mathcal{F}_{q, r}$ arguments $z$ and $a$ by an amount $\lambda$ can be interpreted as a linear finite-Larmorradius correction to the resonance condition (through $\left.z=2(n Y+1) / \beta_{T}^{2}\right)$ and to its width in inhomogenoeus plasmas, through the ratio of Doppler to relativistic width, $a=N_{\|} / \beta_{T}$. The physical meaning of $z \rightarrow z-\lambda$ is the well-known relativistic downshift of the peak $z$ of emission/absorption, which also becomes manifest in a change of width of the emitting/absorbing layer, through $a \rightarrow a-\lambda$. Note that fully and weakly relativistic treatments slightly disagreed on the peak power deposition in some recent ITER modelling ${ }^{27}$ and that the present $z$ shift goes in the right direction to reconcile this disagreement. 


\section{COMPARISON WITH OTHER DISPERSION FUNCTIONS}

Table II clarifies under which conditions $\mathcal{F}_{q, r}(n)$ reduces to other semi-relativistic and classical plasma dispersion functions.

The $\lambda /(1-i t) \simeq \lambda$ approximation of Eq.29 quoted in the table is equivalent to zeroing the $\lambda$ shifts in Eq.31. The limit is justified under very weakly relativistic conditions, such that $t=\beta_{T}^{2} \tau \ll 1$ for any "reasonable" $\tau$, in the sense of Subsec.II.A.

We also note that double sums over the Shkarofsky function index $m$ (sometimes called $q$ or $q+1 / 2$ or $p+n+3 / 2$ in literature) and over the harmonic number $n$ were already found by Airoldi ${ }^{15}$, Shkarofsky ${ }^{7}$ and Swanson ${ }^{10,28}$.

\section{A. Shkarofsky's Tensor}

Eqs.28 and 35-40 generalize the well-known Shkarofsky's weakly relativistic tensor ${ }^{3}$ by

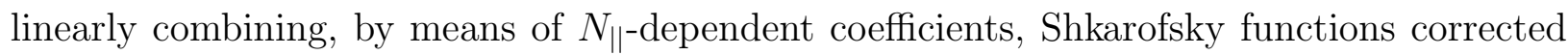
for FLR effects. Higher $\beta_{T}$ orders are also retained, compared to Shkarofsky's treatment.

The original tensor ${ }^{3}$ can be obtained as follows. In the $a$ and $b$ coefficients (Eqs.42-45) one has to a) keep only the lowest order in $\beta_{T}$, b) set $e^{-\lambda}=1$ and c) $N_{\|}=0$. Approximations b) and c) are equivalent to neglect finite Larmor radius and oblique incidence corrections, respectively. As a result, the only non-vanishing coefficients are $a_{5}=\beta_{T}^{3}$ and $b_{7}=\beta_{T}^{5}$. Correspondingly, only the $\mathcal{F}_{n+\frac{5}{2}}$ and $\mathcal{F}_{n+\frac{7}{2}}$ functions remain in use and Eqs.35-40 simplify as follows:

$$
\begin{aligned}
\mathcal{Q}_{m n, 11} & =\mathcal{Q}_{m n, 22}=\frac{i \beta_{T}^{3}}{2} \mathcal{F}_{m+\frac{5}{2}}^{+, 1} \\
\mathcal{Q}_{m n, 12} & =\frac{\beta_{T}^{3}}{2} \mathcal{F}_{m+\frac{5}{2}}^{-, 1} \\
\mathcal{Q}_{m n, 13} & =-i N_{\|} \frac{N_{\perp}}{Y} \frac{\beta_{T}^{3}}{2} \mathcal{F}_{m+\frac{7}{2}}^{-, 1}{ }^{\prime} \\
\mathcal{Q}_{m n, 23} & =N_{\|} \frac{N_{\perp}}{Y} \frac{\beta_{T}^{3}}{2}\left(\mathcal{F}_{m+\frac{7}{2}}^{+, 1}{ }^{\prime}-2 \mathcal{F}_{m+\frac{7}{2}}^{\prime}\right) \\
\mathcal{Q}_{m n, 33} & =i \beta_{T}^{3} \mathcal{F}_{m+\frac{5}{2}}+i N_{\|}^{2} \beta_{T} \mathcal{F}_{m+\frac{7}{2}}{ }^{\prime \prime}
\end{aligned}
$$

Moreover, d) the FLR parameter $\lambda$ has to be zeroed in Eq.31, e) the second sum in Eq.28 has to be truncated to $m=|n|$,

$$
\epsilon_{i j}=\delta_{i j}+\frac{i X}{\beta_{T}^{5}}\left[\mathcal{Q}_{00, i j}+\sum_{n=1}^{\infty} \frac{\lambda^{n}}{2^{n} n !}\left(\mathcal{Q}_{n n, i j}+\mathcal{Q}_{n-n, i j}\right)\right],
$$


which is equivalent to truncate the Taylor expansion of Bessel functions $I_{n}$ to their leading order, $\lambda^{n}$. Additionally, f) only the leading $\beta_{T}$ order has to be retained at denominator in Eq.28.

Substituting Eqs.46-50 in Eq.51 returns Eqs.4a-4c of Shkarofsky ${ }^{3}$. Note that the 11 and 22 components are identical, while they differ from each other in the fully relativistic tensor ${ }^{1}$, in the present weakly relativistic treatment (Eqs.35 and 36) and in the warm dielectric tensor ${ }^{29}$, as they should in presence of finite but relativistically distorted Larmor orbits, due to the different nature of the Doppler shift (transverse or longitudinal) that breaks the cylindrical symmetry of the plasma response around the magnetic field direction.

\section{B. Warm Tensor}

It is well-known ${ }^{6}$ that Shkarofsky functions can be expressed in terms of the classical dispersion function $Z$. Under proper limits (Tab.I) they actually coincide with it, apart

from a factor: at low $\beta_{T}$, both arguments $z=\frac{1-n Y}{\beta_{T}^{2}}-\lambda$ and $a=\frac{N_{\|}^{2}}{2 \beta_{T}^{2}}-\lambda$ diverge, the $-\lambda$ corrections introduced in Eq.31 become negligible and the asymptotic limit ${ }^{24,30}$

$$
\mathcal{F}_{q}(z, a) \simeq-\frac{Z(\Psi)}{\sqrt{4 a+2 q}} \simeq-\frac{\beta_{T}}{\sqrt{2} N_{\|}} Z(\Psi)
$$

applies. Here

$$
\Psi=\frac{z+q}{\sqrt{4 a+2 q}} \simeq \frac{1}{\sqrt{2}} \frac{1-n Y}{N_{\|} \beta_{T}} .
$$

On the other hand, it is easy to identify the sum over $m$ in Eq.27 as the generating function for the modified Bessel function of the first kind:

$$
\exp \left[\frac{\lambda \cos y t}{1-i t}\right]=\sum_{n=-\infty}^{\infty} I_{n}\left(\frac{\lambda}{1-i t}\right) e^{i n y t}
$$

that, for $\lambda /(1-i t) \simeq \lambda$ yields a familiar portion of the warm dielectric tensor components $\epsilon_{11}, \epsilon_{22}, \epsilon_{33}, \epsilon_{13}:$

$$
\exp \left[\frac{\lambda \cos y t}{1-i t}\right] \simeq \sum_{n=-\infty}^{\infty} I_{n}(\lambda) e^{i n y t}
$$

To recover the other components it is useful to remember the recursive relation for the Bessel function derivative ${ }^{23}$ :

$$
I_{n}^{\prime}=I_{n+1}+n I_{n} / \lambda
$$

After these simple steps the warm dielectric tensor (Eqs.10.40, 57, $58 \mathrm{of}^{29}$ ) is obtained. 


\section{Bessel Functions of complex time-dependent Larmor radius parameter}

Yet another semi-relativistic formulation can be derived by inserting Eq.54, instead of Eq.27, in Eqs.4 and 16. Eq.54 generalizes a standard Bessel function identity utilized in deriving the warm non-relativistic dielectric tensor ${ }^{31}$. However, it does so by replacing $\lambda$ with $\lambda /(1-i t)$, i.e. by introducing a time dependence for $I_{n}$ that cannot be factored out of the time integral anymore. The resulting expression,

$$
\epsilon_{i j}=\delta_{i j}+\frac{i X}{\beta_{T}^{5}+\frac{15}{8} \beta_{T}^{7}} \sum_{n=-\infty}^{\infty} \int_{0}^{\infty} \frac{d t}{\beta_{T}^{2}} Q_{i j} \exp \left[\frac{i t}{\beta_{T}^{2}}(1-n Y)-\frac{\lambda+N_{\|}^{2} t^{2} / 2 \beta_{T}^{2}}{1-i t}\right] I_{n}\left(\frac{\lambda}{1-i t}\right)
$$

avoids the nuisance of the double sum over $m$ and $n$ but involves integrals more complicated than Eq.29 and is not convenient from a numerical standpoint. Actually it exceeds in complexity the initial fully relativistic Trubnikov's tensor of Eqs.4 and 11. Nevertheless, it is useful as it links the present weakly relativistic treatment to others. For example, an equation similar to Eq.57 was the starting point for the original Shkarofsky's derivation of $1966^{3}$ and for a revised, more general result of $1986^{7}$. While the Bessel functions were approximated to their leading order in $\lambda$ in the initial derivation, the whole series of powers of $\lambda$ was retained in the later work, yielding a double sum similar to Eq.28, the other sum being on the harmonic number. Also Eqs.2.3.53 and 78 of $^{14}$ share interesting analogies with Eq.57 of the present article, in spite of the different method. There are also distinct differences, though, including the argument of the Bessel function, which was $\lambda$ in those previous works ${ }^{3,7,14}$, or the more drastic simplification of $Q_{i j}$, on the ground that the exponent has a bigger effect on the integral in Eq.57.

At this point it should be remembered that it is customary to treat as a constant the Lorentz factor $\gamma$ in the denominator in the integrand of Eq.5 and to Taylor-expand only the factor $\gamma$ in the exponent, the reason being that relativistic corrections in the denominator have a comparatively small effect on the integral. This is equivalent to approximating $Q_{i j} \simeq$ $\left(T_{i j}^{(1)} 2 b-T_{i j}^{(2)}\right) 8 b^{2}$, which in turn leads to the loss of $N_{\|}$corrections in Eqs.35-45. Probably

this was oversighted in the past due to the quasi-perpendicular hypothesis $N_{\|} \ll \beta_{T}$, which tended to neglect small- $N_{\|}$corrections when summed to or divided by (comparativley larger) $\beta_{T}$ corrections. 


\section{NUMERICAL RESULTS}

The double sum in Eq.28 may seem computationally expensive, but only four functions $\mathcal{F}_{m+\frac{l}{2}}$, with $l=5,7,9$ and 11 , are used at each step $m$ and only two of them need to be calculated. The other two can be recursively generated by ${ }^{6,24}$ :

$$
\frac{N_{\|}^{2}}{\beta_{T}^{2}} \mathcal{F}_{m+\frac{5}{2}}(n)=1+\frac{2}{\beta_{T}^{2}}\left(N_{\|}^{2}-1+m Y\right) \mathcal{F}_{m+\frac{1}{2}}(n)-\left(m+\frac{1}{2}\right) \mathcal{F}_{m+\frac{3}{2}}(n)
$$

The same relation also provides the functions for the following step, $m+1$, and so on. Hence, multiple values of $m$ in the sum of Eq.28 do not require significant extra calculations compared to the single value, $m=|n|$ case of Shkarofsky ${ }^{3}$.

The sum over $n$ doesn't introduce significant calculations either, because the generic step $n$ involves functions that are either known from steps $n-1$ and $n-2$, or will be reutilized for the $n+1$ - and $n+2$-th harmonic. Furthermore, unless $\beta_{T}$ and/or $N_{\|}$are high enough to cause significant harmonic overlap, i.e. if the electron cyclotron lines, although broadened, are still well-resolved, the double sum can be restricted to few values of $n$ or just to the dominant harmonic.

The index $m$ can also be kept small, similar to $k \leq 3$ of Swanson's moderately relativistic dielectric tensor ${ }^{28}: m=2$ is typically a good compromise between accuracy and rapidity of calculation.

Two applications are considered in the following Subsections, to illustrate the advantages of Eq.28 over the cold and warm dielectric tensor ${ }^{29}$ as well as the semi-relativistic formulations by Shkarofsky ${ }^{3}$ and Airoldi-Orefice ${ }^{15}$. To author's knowledge, the latter is computed for the first time in its generality: even the original article, despite the generality of the analytical derivation, treated numerically only a simplified case, virtually coincident with Shkarofsky's tensor. The models are compared with each other and with Trubnikov's tensor ${ }^{1}$ (from which they all descend, after various approximations), which is taken as a reference.

\section{A. OXB Mode Conversion}

An interesting application for the new tensor is represented by the dispersion relation $\operatorname{det}\left(N_{i} N_{j}-N^{2} \delta_{i j}+\epsilon_{i j}\right)=0$ for an optimal value of the parallel refractive index, $N_{\|}=$ $\sqrt{Y /(Y+1)}$, enabling the ordinary $(\mathrm{O})$-extraordinary $(\mathrm{X})$-Bernstein $(\mathrm{B})$ mode conversion ${ }^{32}$. 
For heating or emission measurements at a low harmonic $\left(\omega=n \omega_{c}\right.$, with $\left.n=1-5\right)$, the optimal parallel component for the mode conversion takes values $N_{\|} \simeq 0.4-0.7$. These are incompatible with quasi-perpendicular incidence $\left(N_{\|} \ll \beta_{T}\right.$, Eq.1) and thus not adequately modelled by Shkarofsky's and other mild relativistic formulas, and constitute a good testbed for the new results. A slab geometry is considered for simplicity. Note, however, that in more realistic geometries $N_{\|}$tends to grow even larger than the initial $N_{\|} \simeq 0.4-0.7$, as a result of the large $N_{\perp}$ being projected in a curved magnetic field. This represents an additional argumentation in favor of weakly relativistic formulas valid for arbitrary $N_{\|}$.

The dispersion relation was calculated for optimal launch in a uniformly magnetized plasma $(Y=0.9)$ of inhomogeneous density $X$. The contours corresponding to $\operatorname{det}\left(N_{i} N_{j}-\right.$ $\left.N^{2} \delta_{i j}+\epsilon_{i j}\right)=0$ are plotted in Figs.1-5, for different temperature regimes. Although the root finder searched for $N_{\perp}^{2}$ roots, is plotted on the vertical axis, to magnify the mode conversion region at relatively low $N_{\perp}$.

The models examined in Figs.1-5 offer the advantage, over electrostatic approximations ${ }^{11-13}$, of dealing simultaneously with the electromagnetic $\mathrm{O}$ - and X-mode as well as with the electrostatic Bernstein (B) mode.

Apart from the cold dispersion, which does not account for the Bernstein mode and for its generation at the upper hybrid resonance (UHR), and the Shkarofsky tensor, which tends to overestimate $N_{\perp}$ for the B-mode, all the tensors considered are all also very general with respect to $N_{\perp}$, at least at non-relativistic temperatures: in Fig. $1, N_{\perp}$ ranges from $N_{\perp}=0$ at the OX mode conversion, for which the cold dielectric tensor would be sufficient, to $N_{\perp}>1$ at the XB mode conversion, where FLR effects play a fundamental role and the warm dielectric tensor becomes necessary, up to $N_{\perp} \approx 1 / \beta_{T} \gg 1$, for the Bernstein mode.

Most models, including the new one, agree with the fully relativistic reference up to fairly large $N_{\perp}$, but the warm plasma tensor shows good agreement over the broadest domain and yet it is the most economical in terms of computational resources. This confirms the appropriatenes of adopting the warm tensor to trace OXB mode-converted Electron Bernstein Waves (EBWs) in plasmas of $1 \mathrm{keV}$ or $\operatorname{less}^{18}$.

At higher temperatures $\left(\beta_{T}=0.1\right)$ relativistic effects start to become important and only two tensors (the warm one, and the new one presented here) show good agreement with the fully relativistic curve (Fig.2).

Relativistic corrections clearly become important at $\beta_{T}=0.2$, corresponding to 
$T_{e}=20.5 \mathrm{keV}$, as it is evident from the difference between the fully relativistic and the classical 'warm' curve in Fig.3. The new tensor best approximates Trubnikov's results (Fig.3) and it remains the best approximation at up to $\beta_{T}=0.5\left(T_{e}=128 \mathrm{keV}\right.$, Fig.4). The first significant discrepancies are encountered at $\beta_{T}=0.6$ (Fig.5), and can be explained with both the $\beta_{T} \ll 1$ and $\frac{N_{\perp}}{Y} \ll \frac{1}{\beta_{T}^{2}}$ limits being violated. The latter translates in $N_{\perp} \ll 2.5$ in the case of interest, and is responsible for the change of slope at $N_{\perp} \simeq 1.2$.

It is evident from the vertical axes of Figs.1-5 that for EBWs $N_{\perp}$ scales as $1 / \beta_{T}{ }^{33}$. In the light of this, the inequality $\frac{N_{\perp}}{Y} \ll \frac{1}{\beta_{T}^{2}}$ rewrites $\beta_{T} \ll Y$, which in fact is violated in Fig.5.

Finally, note that relativistic effects act in the correct direction, of reducing the perpendicular phase velocity $c / N_{\perp}$ with respect to warm plasma calculations. This is in agreement with the relativistically increased mass and the consequent reduced thermal velocity of electrons, compared to a classical calculation for the same temperature.

Fig.6a shows a detail of Fig.3 -although with $N_{\perp}^{2}$, rather than $N_{\perp}$, on the vertical axis- in the vicinity of the OX mode conversion: the O-mode branch comes from low densities and connects to the SX-mode branch at the cutoff density. Two main conclusions can be drawn from this plot.

First of all, the weakly and fully relativistic O-mode penetrates deeper than the cold and warm one. This is in agreement with the mass increase and consequent reduction of the effective plasma frequency, so that the condition $\omega=\omega_{p, r e l}$, where $\omega$ is the wave frequency and $\omega_{p, r e l}$ the relativistically corrected plasma frequency is achieved at slightly higher (normalized) density $X=\omega_{p} / \omega$, i.e. deeper in the plasma ${ }^{34}$.

Secondly, although all drawn for the nominal optimal $N_{\|}$, some curves access the evanescent, $N_{\perp}^{2}<0$ half-plane, indicating an incomplete mode conversion, subject to losses. Slightly different $N_{\|}$settings are necessary for these curves to fully develop in the $N_{\perp}^{2} \geq 0$ half-plane, without accessing the evanescent region (Fig.6b). In other words, different tensors predict different optimal angles for the OX mode conversion, with variations as large as $\pm 2^{\circ}$ for $\beta_{T}=0.2$. The fully relativistic calculation, which is the most reliable, confirms the cold estimate $N_{\|}=\sqrt{Y /(Y+1)}$ to be optimal. This is not surprising because the cold tensor contains the basic physical ingredients for the mode conversion, and relativistic corrections displace both the $\mathrm{O}$ - and the X-mode cutoff density by the same amount: although for the same $N_{\|}$, for $\beta_{T}=0.2$ the conversion takes place at $X=1.05$ rather than at $X=1$.

The new tensor exhibits the best agreement with Trubnikov's reference curve, both with 
respect to the optimal $N_{\|}$and to the cutoff density $X$.

\section{B. Power absorption for oblique incidence}

Here the absorption of electromagnetic waves polarized in the O-mode is studied for various angles of incidence on the fundamental cyclotron resonance, in a moderately relativistic regime $\left(\beta_{T}=0.15\right)$.

The absorption coefficient $\alpha$, defined as the fractional power loss $d P / P$ per unit length $d s$ along the ray $(d P / P=\alpha d s)$, is given by ${ }^{14}$ :

$$
\alpha=2 \frac{E_{i}^{*} \epsilon_{i j}^{a} E_{j}}{\left|E_{i}^{*} \frac{\partial \Lambda_{i j}^{h}}{\partial \mathbf{k}} E_{j}\right|}
$$

where the superscripts ${ }^{h}$ and ${ }^{a}$ denote respectively the hermitian and anti-hermitian part, $E^{*}$ is the complex conjugate of the electric field $E$ (polarized in the O-mode, in this case) and

$$
\Lambda_{i j}=\epsilon_{i j}+N_{i} N_{j}-N^{2} \delta_{i j}
$$

The absorption coefficient is plotted in Figs.7-9a for a mildly relativistic case $\left(\beta_{T}=0.15\right)$, for three values of $N_{\|}$, ranging from quasi-perpendicular incidence (dominated by relativistic broadening) to oblique incidence (with significant Doppler broadening). The coefficient $\alpha$ is expressed in units of vacuum wavelength $\lambda_{0}$.

The optical depth $\tau=\int_{0}^{s} \alpha\left(s^{\prime}\right) d s^{\prime}$ is also presented in dimensionless units, in Figs.7-9b, in terms of $\lambda_{0}$ and of the lengthscale $R_{0}$ for the inhomogeneity of the magnetic field, $Y=R_{0} / R$, where $R$ is a spatial distance.

The third plot in Figs.7-9 shows the absorption shape factor $\alpha e^{-\tau}$, giving the shape of the deposition profile. It should be emphasized that Figs.7-9 refer to a single ray in a slab, hence the deposition width might be underestimated compared to experiments or to more sophisticated modelling of a beam or bundle of rays in a realistic geometry. The main purpose of these plots is to compare various absorption models under the assumptions $R_{0}=1 \mathrm{~m}$ and $\lambda_{0}=2 \mathrm{~mm}$.

The absorption curves obtained with the new tensor agree with other weakly relativistic approximations, in particular with Shkarofsky's, at small-to-intermediate parallel refractive indices, $N_{\|} \leq \beta_{T}$ and show the best agreement with Trubnikov's fully relativistic absorption 
profiles for large angles of propagation relative to the magnetic field, such that $N_{\|}>\beta_{T}$. This is best exemplified by the optical depth $\tau$ in Fig.9b. Potential applications include problems requiring the power localization to be so accurate that small differences between the weakly and fully relativistic predictions do matter, as in the stabilization of neoclassical tearing modes in $\mathrm{ITER}^{27}$.

\section{Computational time}

Table III summarizes the time necessary for calculating the curves in Fig.3 for the choice of parameter shown.

Evaluating the new tensor was slightly slower than computing Shkarofsky's tensor, but orders of magnitude faster than the fully relativistic treatment. This may be convenient for massive repetitive calculations: the curves in question feature only 10000 points each, but the dielectric tensor may need to be evaluated up to $10^{8}$ times in tracing 1-10 bundles of 10-100 rays, modelled by up to 10000 points each (their spacing being smaller than the plasma size and the plasma inhomogeneity length-scale, but large compared to the wavelength, which for large refractive indices can become very small). Such an estimate also takes into account that the tensor may need to be evaluated $\sim 10$ times for each spatial step, for example if a 4-5th order Runge-Kutta time-advance scheme is adopted, and derivatives are needed, which in turn require the tensor to be evaluated in displaced points.

The scaling of the computational time with the parameters is also reported in Table III, allowing extrapolations to different parameter settings. The accuracy of the results

depends in a more complicated manner upon the parameter settings. However, although the considered expressions formally involve infinite sums, it was empirically found that small truncation indices are generally sufficient. On the other hand modest improvements, and at higher and higher computational costs, are registered if the parameters are set to values larger or much larger than those in Table III.

The original notations have been preserved throughout the table. Most parameters, like $k_{\text {max }}$ and $m$, are truncation indices for power series (finite Larmor radius expansions) or, like $q_{\max }$ and $n$, for sums over Shkarofsky or Bessel functions (cyclotron harmonic expansions). In the slightly more complicated expression of Ref. ${ }^{15}, \sigma$ and its combinatorics decompositions control the approximation of the exponent; $m$ is an index for a generalized Shkarofsky 
function of $q=0$ and $r \neq 0$, expanded in Gamma-functions and binomials; $k$ is a truncating index for the expansion of the Bessel functions in the FLR parameter; $n$ is the harmonic number; $j$ and $L$ are relevant to two binomial expansions operated in the referenced article.

The integral in the second formula by Trubnikov ${ }^{1}$, reported for convenience in Eqs.4, 11, was numerically integrated. In principle the domain of integration extends from $\tau=0$ to $\tau=\infty$, but 400 gyroperiods were retained for the numerical integration and discretized in $n_{\tau}=20000$ intervals to study the dispersion (Subsec.V A), while 800 gyroperiods discretized in $n_{\tau}=80000$ steps were used to study the absorption (Subsec.V B). The finer resolution was necessary to avoid unphysically multi-peaked deposition profiles.

Finally, high Shkarofsky function indices $q$ are needed, in general, in order to accurately model the dispersion and absorption at large $N_{\perp}$, but the new tensor converges more rapidly in $m$. Yet, with $m=2$, it reproduces Trubnikov's results better than Shkarofsky does for $q=7 / 2$, which corresponds to $m=3$.

\section{SUMMARY AND CONCLUSIONS}

In summary a novel formulation of the semi-relativistic dielectric tensor, Eq.28, was derived in the present article in the form of a power series in the Larmor radius parameter $\lambda$, converging for any perpendicular refractive index $N_{\perp} \ll Y / \beta_{T}^{2}$, of a strictly weakly relativistic approximation $\left(\beta_{T} \ll 1\right)$.

Its generality and complexity are intermediate between Trubnikov's fully relativistic tensor and Shkarofsky's weakly relativistic tensor, in that it is also weakly relativistic, but does not contain approximations on $a=N_{\|} / \beta_{T}$ nor on the Larmor radius parameter $\lambda$, except for those which indirectly follow from $\beta_{T} \ll 1$ and from the factorization of an exponent in the time integral, based on the relatively benign request of Eq.19.

The new tensor describes the propagation (including electrostatic propagation based on finite Larmor radius effects) and damping (corrected for relativistic effects and Doppler broadening) of modes of arbitrary wavenumbers and orientation relative to the magnetic field, including the Bernstein mode. The new tensor was tested in and applied to calculations of Doppler and relativistic broadening as well as in the dispersion relation for the OXB mode conversion. 


\section{Acknowledgments}

The author thanks N.Marushchenko and R.Prater for the encouragement and the stimulating discussions.

\section{APPENDIX: DERIVATION OF EQS.35-40}

In order to identify $\mathcal{F}_{q, r}$ functions in Eq.29, it is useful to define two operators in analogy with $T^{(1)}$ and $T^{(2)}$ of Eqs.6, 14:

$$
\begin{aligned}
& \mathcal{T}^{(1)}=\left(\begin{array}{ccc}
C & -S & 0 \\
S & C & 0 \\
0 & 0 & I
\end{array}\right) \\
& \mathcal{T}^{(2)}=\frac{1}{Y^{2}}\left(\begin{array}{ccc}
N_{\perp}^{2} S^{2} & N_{\perp}^{2} S(C-1) & N_{\perp} N_{\|} S L \\
-N_{\perp}^{2} S(C-1) & -N_{\perp}^{2}(C-1)^{2} & -N_{\perp} N_{\|}(C-1) L \\
N_{\perp} N_{\|} S L & N_{\perp} N_{\|}(C-1) L & N_{\|}^{2} L^{2}
\end{array}\right)
\end{aligned}
$$

These are indeed matrices of operators. The individual operators are $C, S, L$ and $I$. They act on an integral by multiplying its integrand by, respectively, $\cos y t, \sin y t, y t$ or 1 . Their effect on generalized Shkarofsky functions is simply to change the harmonic number $n$ or the index $r$ :

$$
\begin{aligned}
C \mathcal{F}_{q, r} & =\left[\mathcal{F}_{q, r}(n+1)+\mathcal{F}_{q, r}(n-1)\right] / 2=\mathcal{F}_{q, r}^{+, 1} / 2 \\
S \mathcal{F}_{q, r} & =\left[-\mathcal{F}_{q, r}(n+1)+\mathcal{F}_{q, r}(n-1)\right] / 2 i=-\mathcal{F}_{q, r}^{-, 1} / 2 i \\
L \mathcal{F}_{q, r} & =-i y \mathcal{F}_{q, r+1} \\
I \mathcal{F}_{q, r} & =\mathcal{F}_{q, r}
\end{aligned}
$$

Here, when the argument $n$ is dropped, it is understood that $\mathcal{F}_{q, r}$ refers to the $n$-th harmonic. The apices in Eqs.A.3-A.4 denote a sum or difference of Shkarofsky functions, according to Eq.32. Finally, the $r=0$ case of Eq.A.5 is, according to Eq.33,

$$
L \mathcal{F}_{q}=-i y \mathcal{F}_{q, 1}=-i y\left(\mathcal{F}_{q}-\mathcal{F}_{q-1}\right)=-i y \mathcal{F}_{q}^{\prime}
$$

The new operators allow to handle the numerous integrals of type 29 and to obtain or recognize a new integral by applying an operator (i.e., changing the indices) to an old 
one. With this approach, all integrals descend from one. This initial integral, $i \frac{e^{-\lambda}}{\beta_{T}^{2}} \mathcal{F}_{m, 0}$, is obtained from Eq.29 for $Q_{i j}=1$. Following the scheme of Eqs.18 it is easy to combine the above operators into another one, that multiplies integrands by $Q_{i j}$. Then Eq.29 becomes:

$$
\begin{aligned}
\mathcal{Q}_{m n, i j}= & i e^{-\lambda} \quad \beta_{T}^{3} \mathcal{T}_{i j}^{(1)}\left[\mathcal{F}_{m+\frac{5}{2}}+\frac{15}{8} \beta_{T}^{2} \mathcal{F}_{m+\frac{7}{2}}+\frac{5}{4} N_{\|}^{2} \mathcal{F}_{m+\frac{9}{2}, 2}-\frac{5}{4} \beta_{T}^{2} \lambda\left(2 \mathcal{F}_{m+\frac{9}{2}}-\mathcal{F}_{m+\frac{9}{2}}^{+, 1}\right)\right] \text { (A.8) } \\
& -i e^{-\lambda} \beta_{T}^{5} \mathcal{T}_{i j}^{(2)}\left[\mathcal{F}_{m+\frac{7}{2}}+\frac{35}{8} \beta_{T}^{2} \mathcal{F}_{m+\frac{9}{2}}+\frac{7}{4} N_{\|}^{2} \mathcal{F}_{m+\frac{11}{2}, 2}-\frac{7}{4} \beta_{T}^{2} \lambda\left(2 \mathcal{F}_{m+\frac{11}{2}}-\mathcal{F}_{m+\frac{1}{2}}^{+, 1}(\mathrm{~A}) \cdot\right] 9\right)
\end{aligned}
$$

This contains some generalized functions, $\mathcal{F}_{m+\frac{9}{2}, 2}$ and $\mathcal{F}_{m+\frac{11}{2}, 2}$, that can be reconducted to simple Shkarofsky functions of $r=0$ by integrating by parts (Eq.34):

$$
\mathcal{Q}_{m n, i j}=i \mathcal{T}_{i j}^{(1)}\left(a_{l} \mathcal{F}_{m+\frac{l}{2}}+a_{+} \mathcal{F}_{m+\frac{9}{2}}^{+, 1}\right)-i \mathcal{T}_{i j}^{(2)}\left(b_{l} \mathcal{F}_{m+\frac{l}{2}}+b_{+} \mathcal{F}_{m+\frac{11}{2}}^{+, 1}\right)
$$

where the convention of summing over repeated indices is adopted and the coefficients $a$ and $b$ were given in Eqs.42-45.

Applying $\mathcal{T}^{(1)}$ and $\mathcal{T}^{(2)}$ does not rise the index $r=0$ of the $\mathcal{F}_{q, r}$ functions in Eq.A.10, because neither of the operators that they contain acts on $r$ (see Eqs.A.3, A.4 and A.7) ${ }^{37}$. Also, they don't introduce any new explicit dependence on $\beta_{T}$.

At this point $\mathcal{T}_{i j}^{(1)}$ and $\mathcal{T}_{i j}^{(2)}$ should be detailed according to Eqs.A.1-A.2. For instance, for the first component,

$$
\mathcal{Q}_{m n, 11}=i C\left(a_{l} \mathcal{F}_{m+\frac{l}{2}}+a_{+} \mathcal{F}_{m+\frac{9}{2}}^{+, 1}\right)-i \frac{N_{\perp}^{2}}{Y^{2}} S^{2}\left(b_{l} \mathcal{F}_{m+\frac{l}{2}}+b_{+} \mathcal{F}_{m+\frac{11}{2}}^{+, 1}\right)
$$

Following the rules for $C$ (Eq.A.3) and $S$ (Eq.A.4), the result Eq.35 is found. The same procedure can be used for the other components, leading to Eqs.36-40. 
* volpe@fusion.gat.com; Present address: General Atomics, San Diego, CA, U.S.A.

1 B. A. Trubnikov, in Plasma Physics and the Problem of Controlled Thermonuclear Reactions (Pergamon Press, New York, 1959), vol. III, p. 122.

2 Y. N. Dnestrovskii, D. P. Kostomarov, and N. V. Skrydlov, Sov. Phys.-Tech. Phys. 8, 691 (1964).

3 I. P. Shkarofsky, Phys. Fluids 9, 561 (1966).

4 P. A. Robinson, J. Plasma Phys. 37, 435 (1987).

5 P. A. Robinson, J. Plasma Phys. 37, 449 (1987).

6 V. Krivenski and A. Orefice, J. Plasma Phys. 30, 125 (1983).

7 I. P. Shkarofsky, J. Plasma Phys. 35, 319 (1986).

8 P. H. Yoon and R. C. Davidson, J. Plasma Phys. 43, 269 (1990).

9 P. A. Robinson, Phys. Fluids 31, 107 (1988).

10 D. G. Swanson, Plasma Phys. Control. Fusion 44, 1329 (2002).

11 A. N. Saveliev, Plasma Phys. Control. Fusion 47, 2003 (2005).

12 A. D. Piliya, A. Y. Popov, and E. N. Tregubova, Plasma Phys. Control. Fusion 45, 1309 (2003).

13 E. Lazzaro and A. Orefice, Phys. Fluids 23, 2330 (1980).

14 M. Bornatici, R. Cano, O. D. Barbieri, and F. Engelmann, Nucl. Fusion 23, 1153 (1983), sec.2.3.

15 A. C. Airoldi and A. Orefice, J. Plasma Phys. 27, 515 (1982).

16 H. P. Laqua, V. Erckmann, H. J. Hartfuß, H. Laqua, W.-A. Team, and E. Group, Phys. Rev. Lett. 78, 3467 (1997).

17 H. P. Laqua, H. J. Hartfuß, and W.-A. Team, Phys. Rev. Lett. 81, 2060 (1998).

18 F. Volpe and H. P. Laqua, Rev. Sci. Instrum. 74, 1409 (2003).

19 H. P. Laqua, H. Maassberg, N. B. Marushchenko, F. Volpe, A. Weller, W.-A. Team, W. Kasparek, and E. Group, Phys. Rev. Lett. 90, 75003 (2003).

20 C. B. Forest, P. K. Chattopadhyay, R. W. Harvey, and A. P. Smirnov, Phys. Plasmas 7, 1352 (2001).

21 D. W. Ignat and M. W. Ono, Phys. Plasmas 2, 1899 (1995).

22 E. Mazzucato, Phys. Fluids B 4, 3460 (1992).

23 M. Abramowitz and I. A. Stegun, Handbook of Mathematical Functions (Dover Publications, 
New York, 1974), pp.374-379.

24 P. A. Robinson, J. Math. Phys. 27, 1206 (1986).

25 N. M. Temme, A. E. Sumner, and S. S. Sazhin, Astrophys. and Space Sc. 194, 173 (1992).

26 D. B. Melrose (2006), private communication.

27 W.A.Houlberg, C.Gormezano, J.F.Artaud, E.Barbato, V.Basiuk, A.Becoulet, P.Bonoli, R.V.Budny, L.G.Eriksson, D.Farina, et al., Nucl. Fusion 45, 1309 (2005).

28 D. G. Swanson, Plasma Waves, 2nd Edition (Institute of Physics Publishing, Bristol and Philadelphia, 2003), pp.205-207.

29 T. H. Stix, Waves in Plasmas (AIP, New York, 1992), pp.7-9 and 252-258.

30 C. Maroli and V. Petrillo, Physica Scripta 24, 955 (1981).

31 M. Brambilla, Kinetic Theory of Plasma Waves - Homogeneous Plasmas (Oxford Univ.Press, 1998), p.155.

32 J. Preinhaelter and V. Kopecký, J. Plasma Phys. 10, 1 (1973).

33 F. Volpe, Ph.D. thesis, EMAU University Greifswald (2003).

34 S. Pešić and L. Nikolić, Plasma Phys. Control. Fusion 41, L49 (1999).

35 B. D. Fried and S. D. Conte, The Plasma Dispersion Function (Academic Press, New York, 1961).

36 Similar to Eqs.2.3.23 and 2.3.75-76 and to Eqs.2.3.35 and 2.3.76-77 of Ref. ${ }^{14}$, respectively. $\left|N_{\|}\right| \ll 1$ is needed, according to Ref. ${ }^{14}$, for the second treatment to be valid and equivalent to the first one.

37 Actually in general $L$ does act on $r$ (Eq.A.5), but its effect on $r=0$ is the same as deriving with respect to $z$ (Eq.A.7). 


\section{TABLES}

TABLE I: Summary of approximations on $N_{\|}$and $\lambda$ in available weakly relativistic dielectric tensors. Some power series are truncated (trunc.). Some $N_{\|}$approximations and asymptotic developments for $\lambda \gg 1$ are accompanied by an electrostatic (e.s.) approximation.

\begin{tabular}{lllll}
\hline \hline & $\lambda \ll 1$ & $\lambda \ll 1$ & $\lambda \gg 1$ & $\lambda \gg 1$ \\
& trunc. & & trunc. \\
\hline$N_{\|}=0$ & 4,5 & & & \\
$N_{\|} \ll \beta_{T}$ & 3,6 & $7-10,28$ & $12($ e.s. $),{ }^{8}$ & 13 (e.s.) \\
arbitr. $N_{\|}$ & & 15 & 11 (e.s.) & \\
\hline \hline
\end{tabular}


TABLE II: Classical and weakly relativistic plasma dispersion functions

\begin{tabular}{lll}
\hline \hline Approximations & Function & Ref. \\
\hline none & $\mathcal{F}_{q, r}(n)$ & Eq.31 \\
$\lambda /(1-i t) \simeq \lambda$ & generaliz. Shkarofsky $\mathcal{F}_{q, r}(z, a)$ & 24 \\
$\lambda /(1-i t) \simeq \lambda, r=0$ & Shkarofsky functions $\mathcal{F}_{q}(z)$ & 3 \\
$\lambda /(1-i t) \simeq \lambda, r=0, a=0$ & Dnestrovskii functions $F_{q}$ & 2 \\
$\lambda /(1-i t) \simeq \lambda, r=0 z=0$ & plasma disp. function $Z(\zeta)$ & 35 \\
\hline \hline
\end{tabular}


TABLE III: Time required for computing the dielectric tensor 10000 times on a $1.6 \mathrm{GHz}$ AMD Sempron processor, for various relativistic, weakly relativistic and classical models (see Fig.1)

\begin{tabular}{|c|c|c|c|}
\hline Model & Parameters & CPU time $t(\mathrm{~s})$ & scaling \\
\hline $\operatorname{cold}^{29}$ & & 0.304 & \\
\hline warm ${ }^{29}$ & nr. Bessel functions $n=2$ & 0.447 & $t \propto n$ \\
\hline Shkarofsky $^{3}$ & $q_{\max }=\frac{7}{2}$ & 2.020 & $t \propto\left(q_{\max }-\frac{1}{2}\right)$ \\
\hline Airoldi-Orefice ${ }^{15}$ & $k_{\max }=3, L_{\max }=3, \sigma_{\max }=3, n=2$ & 227.520 & $t \propto k_{\max } L_{\max } \sigma_{\max } n$ \\
\hline Volpe & $m=3, n=2$ & 2.181 & $t \propto m n$ \\
\hline 2nd Trubnikov ${ }^{1}$ & nr. steps in integral, $n_{\tau}=20000$ & 3689.200 & $t \propto n_{\tau}$ \\
\hline
\end{tabular}




\section{FIGURE CAPTIONS}

FIG.1 (color online) (a) Dispersion relation for OXB mode conversion for $Y=\omega_{c} / \omega=0.9$, optimal $N_{\|}=\sqrt{Y /(Y+1)}$ and a moderate electron thermal velocity $\beta_{T}=v_{T} / c=0.05$, according to various relativistic, weakly relativistic and classical dielectric tensors. $X=\omega_{p} / \omega$ is the normalized density. (b) Detail of Fig.1.a for low $N_{\perp}$, showing the fast eXtraordinary (FX) branch of the dispersion relation, the Ordinary (O) one converting into the slow eXtraordinary (SX) which is attracted by the Upper Hybrid Resonance (UHR) and eventually (Fig.1a) converts into the Bernstein (B) mode.

FIG.2 (color online) Like Fig.1a, for $\beta_{T}=0.1$.

FIG.3 (color online) Like Fig.1a, for $\beta_{T}=0.2$.

FIG.4 (color online) Like Fig.1a, for $\beta_{T}=0.5$.

FIG.5 (color online) Like Fig.1a, for $\beta_{T}=0.6$.

FIG.6 (color online) Detail of the dispersion relation for $Y=0.9$ and $\beta_{T}=0.2$ showing the OX mode conversion for (a) $N_{\|}=\sqrt{Y /(Y+1)}=0.688$ and (b) $N_{\|}$individually optimized for each dielectric tensor.

FIG.7 (color online) (a) Absorption coefficient and (b) optical depth in dimensionless units and (c) absorption shape factor for $\beta_{T}=0.15$ and quasi-perpendicular incidence $\left(N_{\|}=0.05\right)$ of O-mode on fundamental electron cyclotron harmonic. $R_{0}=1 \mathrm{~m}, \lambda_{0}=2 \mathrm{~mm}$, $X=0.5$.

FIG.8 (color online) Like Fig.7, except $N_{\|}=0.15$.

FIG.9 (color online) Like Fig.7, but for $N_{\|}=0.45$, making Doppler broadening important. Note the expanded horizontal scale in Fig.c. 

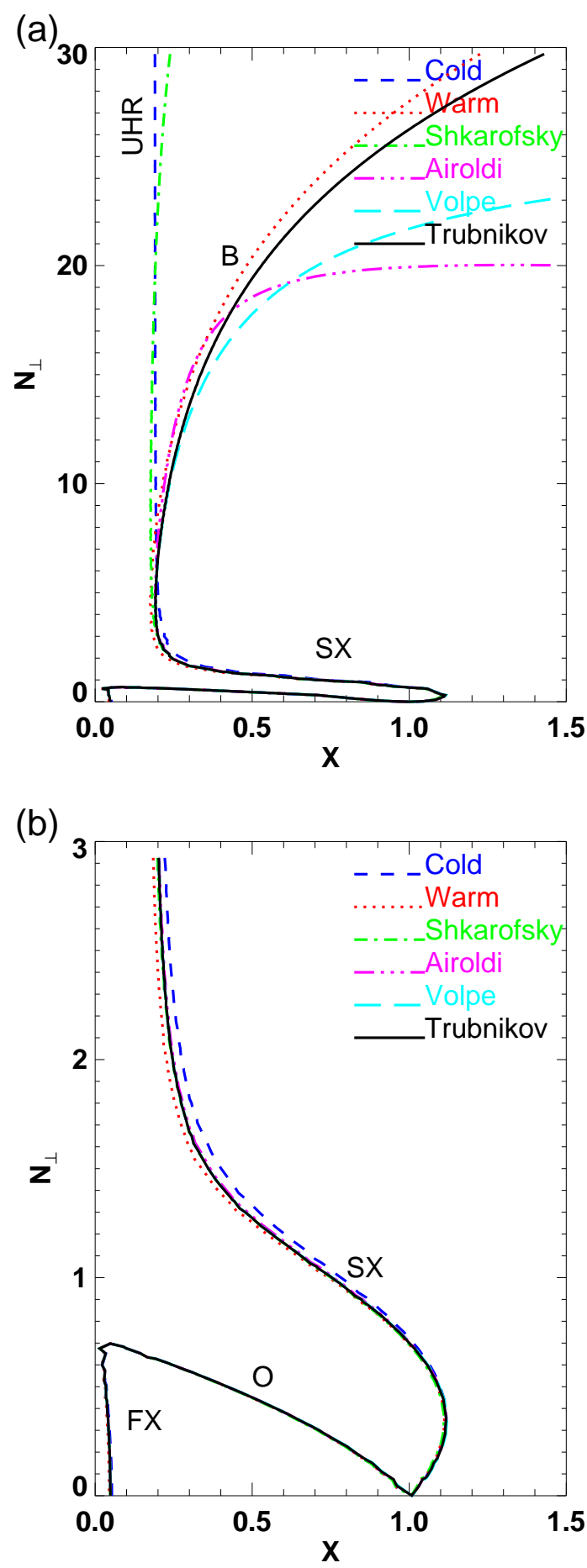

FIG. 1: (color online) (a) Dispersion relation for OXB mode conversion for $Y=\omega_{c} / \omega=0.9$, optimal $N_{\|}=\sqrt{Y /(Y+1)}$ and a moderate electron thermal velocity $\beta_{T}=v_{T} / c=0.05$, according to various relativistic, weakly relativistic and classical dielectric tensors. $X=\omega_{p} / \omega$ is the normalized density. (b) Detail of Fig.1.a for low $N_{\perp}$, showing the fast eXtraordinary (FX) branch of the dispersion relation, the Ordinary $(\mathrm{O})$ one converting into the slow eXtraordinary $(\mathrm{SX})$ which is attracted by the Upper Hybrid Resonance (UHR) and eventually (Fig.1a) converts into the Bernstein (B) mode. 


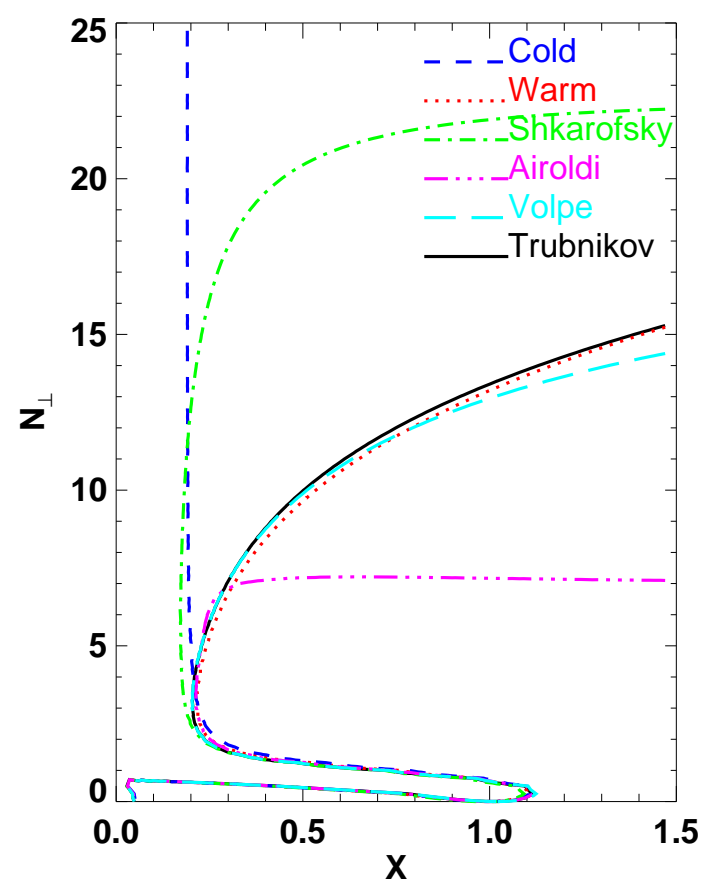

FIG. 2: (color online) Like Fig.1a, for $\beta_{T}=0.1$.

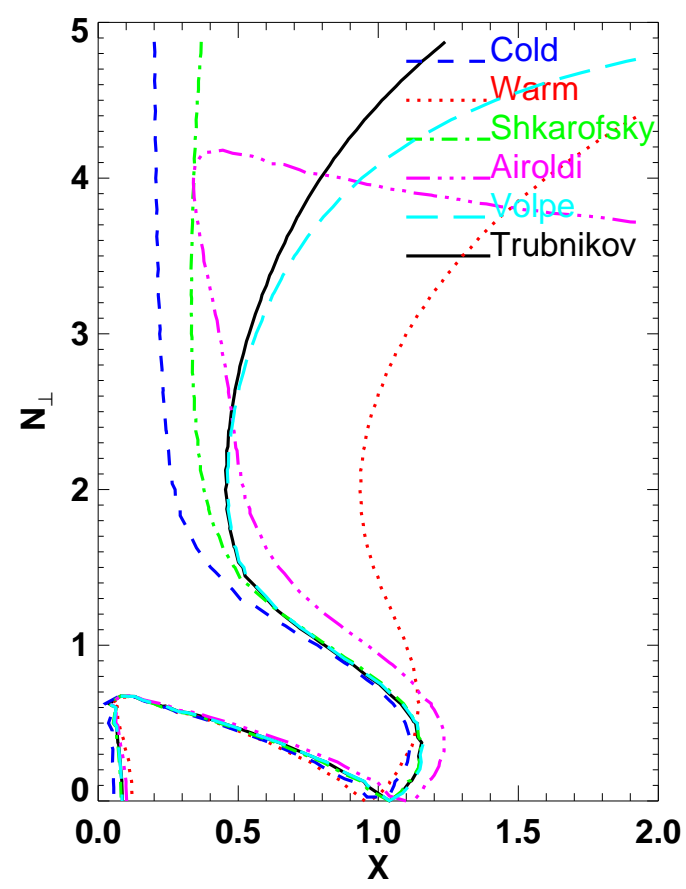

FIG. 3: (color online) Like Fig.1a, for $\beta_{T}=0.2$. 


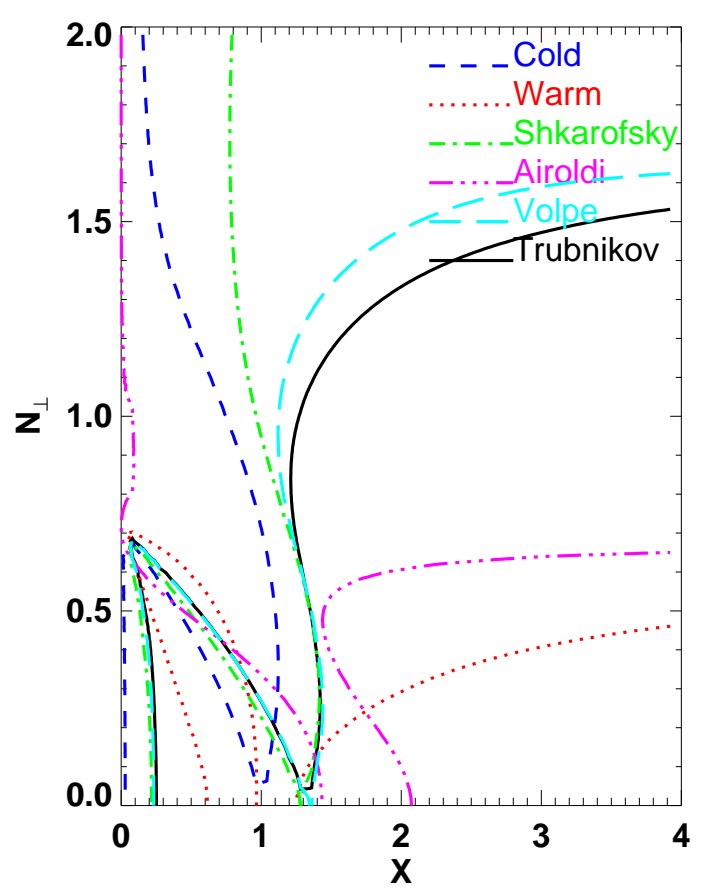

FIG. 4: (color online) Like Fig.1a, for $\beta_{T}=0.5$.

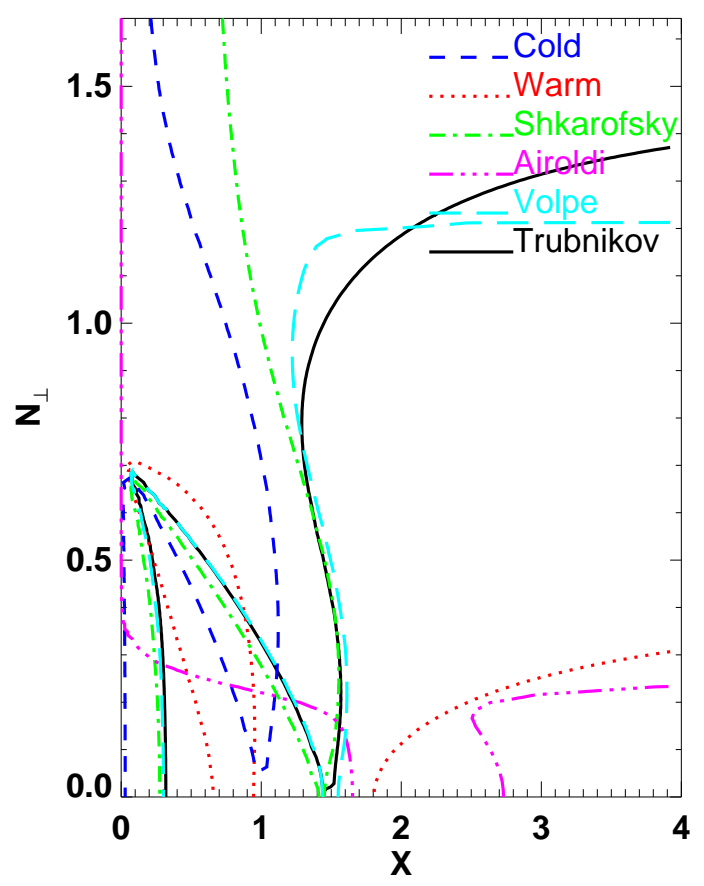

FIG. 5: (color online) Like Fig.1a, for $\beta_{T}=0.6$. 

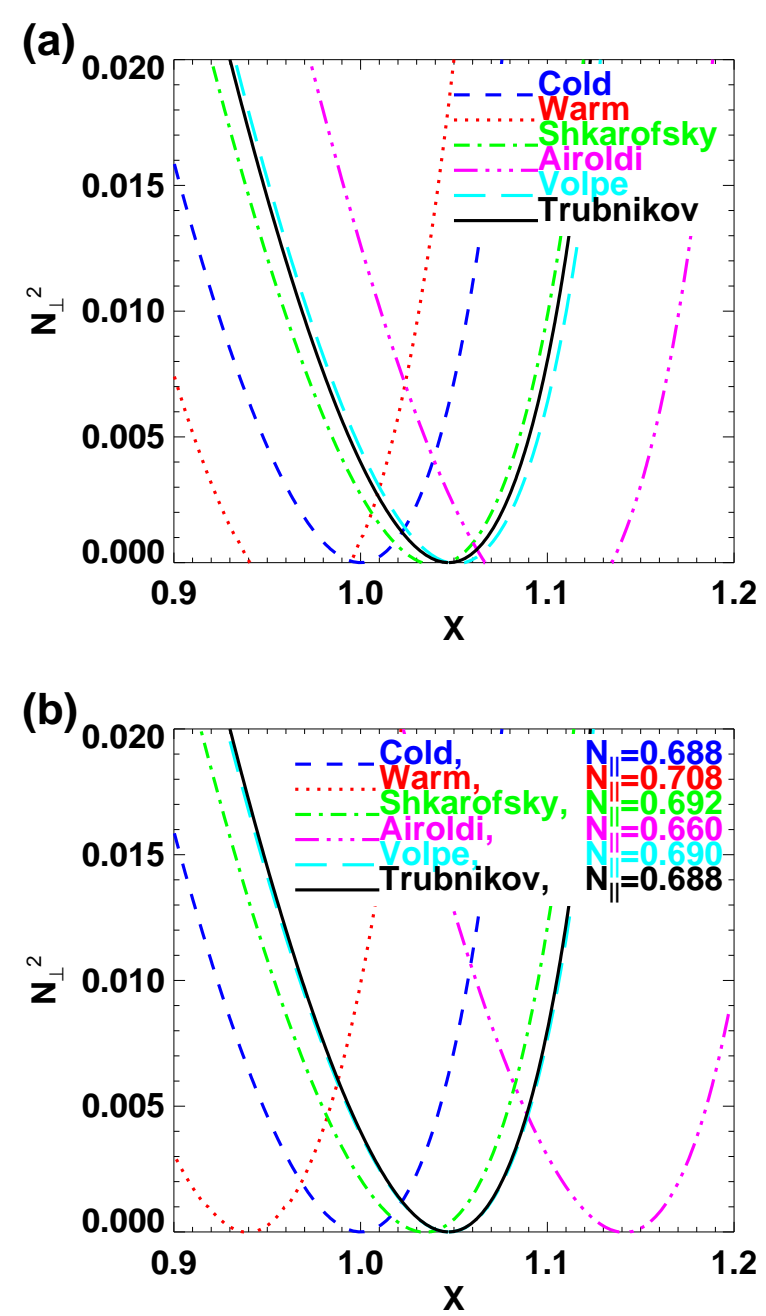

FIG. 6: (color online) Detail of the dispersion relation for $Y=0.9$ and $\beta_{T}=0.2$ showing the OX mode conversion for (a) $N_{\|}=\sqrt{Y /(Y+1)}=0.688$ and (b) $N_{\|}$individually optimized for each dielectric tensor. 


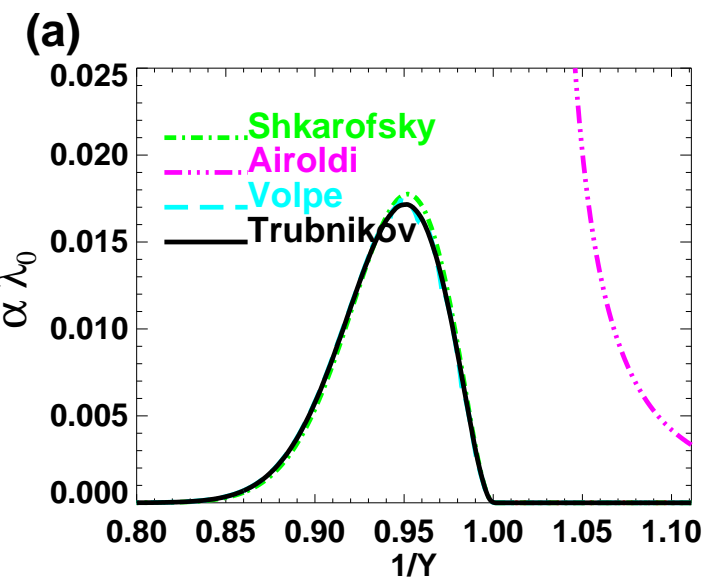

(b)

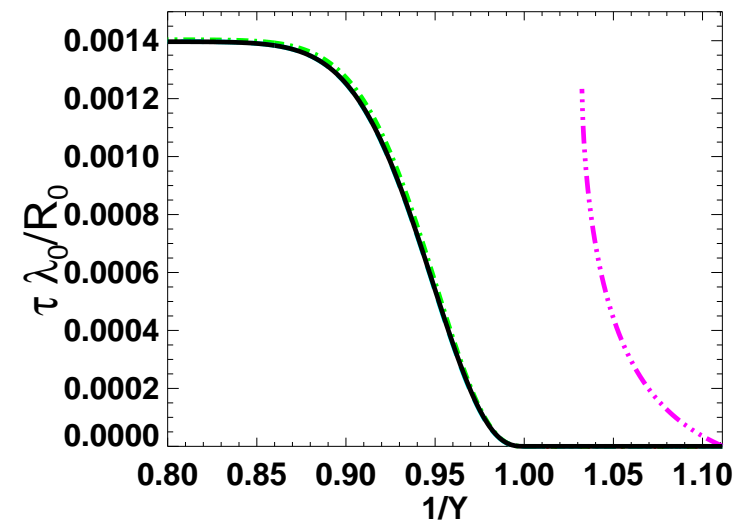

(c)

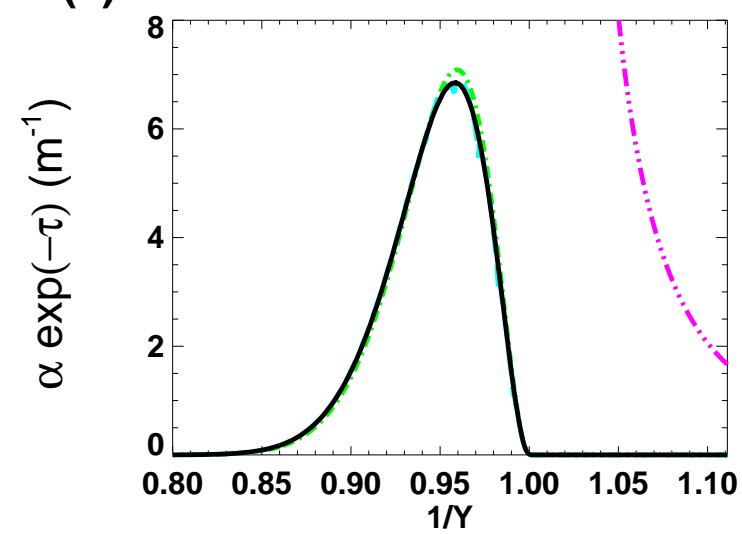

FIG. 7: (color online) (a) Absorption coefficient and (b) optical depth in dimensionless units and (c) absorption shape factor for $\beta_{T}=0.15$ and quasi-perpendicular incidence $\left(N_{\|}=0.05\right)$ of O-mode on fundamental electron cyclotron harmonic. $\mathrm{R}_{0}=1 \mathrm{~m}, \lambda_{0}=2 \mathrm{~mm}, X=0.5$. 


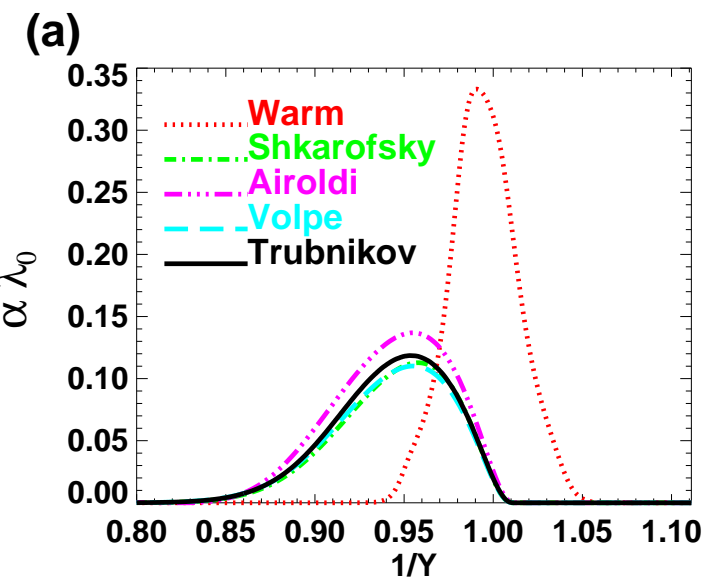

(b)

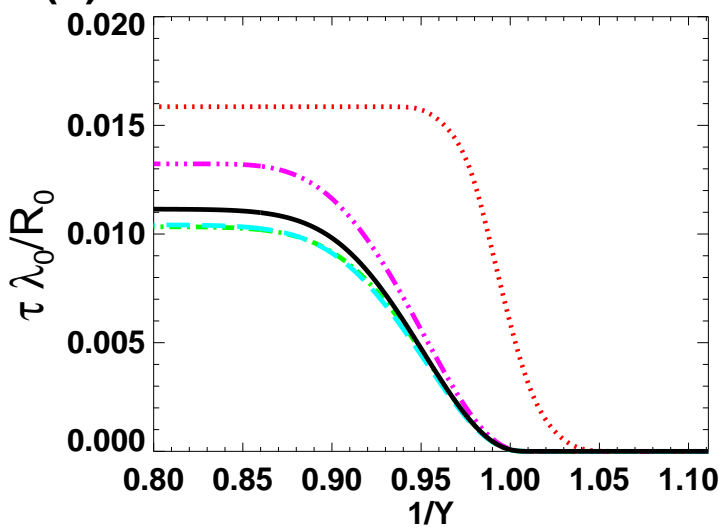

(c)

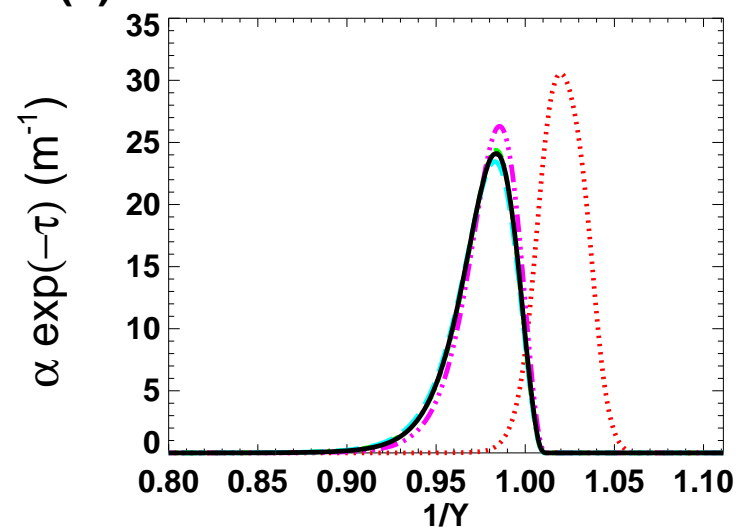

FIG. 8: (color online) Like Fig.7, except $N_{\|}=0.15$. 

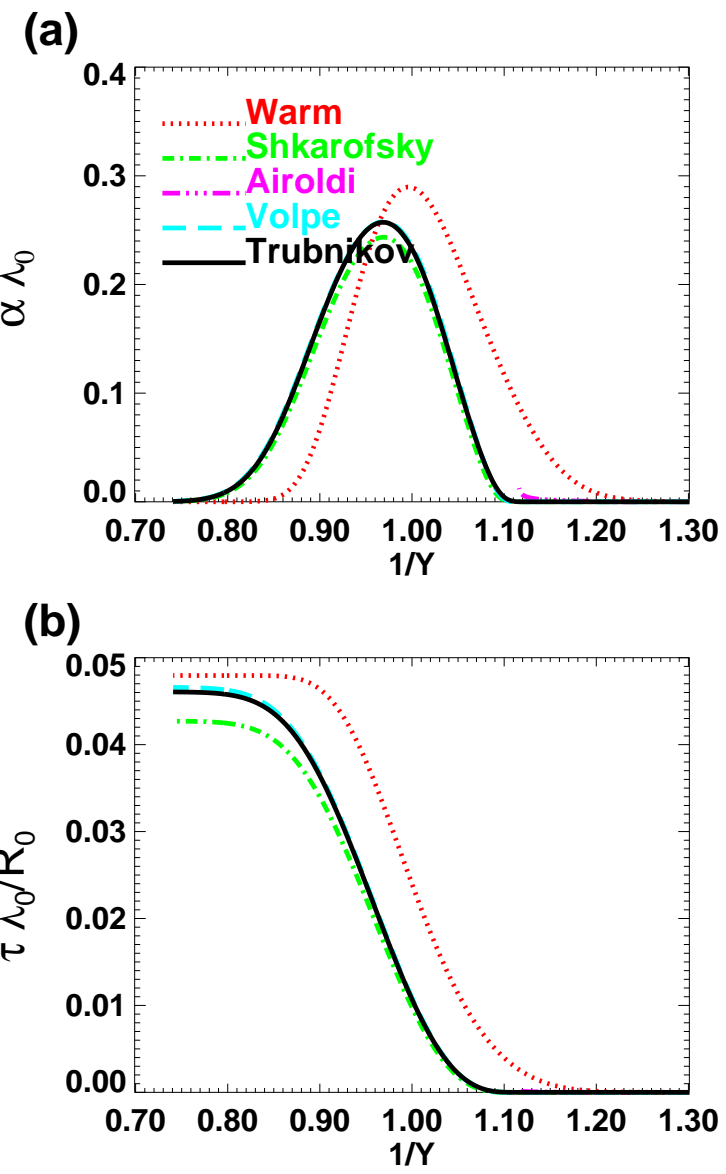

(c)

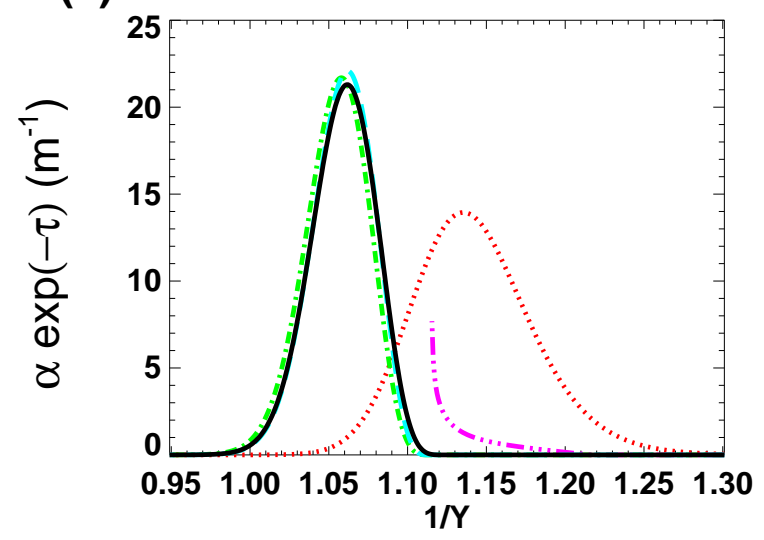

FIG. 9: (color online) Like Fig.7, but for $N_{\|}=0.45$, making Doppler broadening important. Note the expanded horizontal scale in Fig.c. 